\title{
A Quarter Century of Surfactant Research - From Early Neonatal Stabilisation to Long-Term Outcome
}

Proceedings of the 25th International Workshop on

Surfactant Replacement, Moscow, June 10-12, 2010

Guest Editors

Elena Baibarina, Moscow

Tore Curstedt, Stockholm

Henry L. Halliday, Belfast

Mikko Hallman, Oulu

Ola D. Saugstad, Oslo

Christian P. Speer, Würzburg 